\title{
Correction: Using the Patient Portal Sexual Health Instrument in Surveys and Patient Questionnaires Among Sexual Minority Men in the United States: Cross-sectional Psychometric Validation Study
}

Kevon-Mark P Jackman ${ }^{1}$, DrPH, MPH; Jeremy Kane ${ }^{2}$, PhD, MPH; Hadi Kharrazi ${ }^{3}$, PhD, MD; Renee M Johnson ${ }^{1}$, $\mathrm{PhD}, \mathrm{MPH}$; Carl Latkin ${ }^{4}, \mathrm{MS}, \mathrm{PhD}$

\footnotetext{
${ }^{1}$ Department of Mental Health, Johns Hopkins Bloomberg School of Public Health, Baltimore, MD, United States

${ }^{2}$ Department of Epidemiology, Columbia University Mailman School of Public Health, NY, NY, United States

${ }^{3}$ Department of Health Policy and Management, Center for Population Health IT, Johns Hopkins Bloomberg School of Public Health, Batimore, MD, United States

${ }^{4}$ Department of Health, Behavior, and Society, Johns Hopkins Blooomberg School of Public Health, Baltimore, MD, United States
}

\section{Corresponding Author:}

Kevon-Mark P Jackman, DrPH, MPH

Department of Mental Health

Johns Hopkins Bloomberg School of Public Health

624 N Broadway

Hampton House 8th Floor

Baltimore, MD, 21205

United States

Phone: 14109553910

Email: kjackma2@jhmi.edu

\section{Related Article:}

Correction of: https://www.jmir.org/2021/2/e18750/

(J Med Internet Res 2021;23(3):e28358) doi: 10.2196/28358

In "Using the Patient Portal Sexual Health Instrument in Surveys and Patient Questionnaires Among Sexual Minority Men in the United States: Cross-sectional Psychometric Validation Study" (J Med Internet Res 2021;23(2):e18750) four errors were noted.

In the originally published paper, the "greater than or equal to" symbol $(\geq)$ was missing in three places in tables and in one place in the main text due to an XML conversion error. The following corrections have been made:

In Table 1, under "Age (years)," "40" has been corrected to " $\geq 40 "$ ".

In Table 5, under "Age category," "40" has been corrected to " $\geq 40 "$ ".

In Table 6, under "Age category," "40" has been corrected to " $\geq 40 "$ ".
In the section "AMIS-PPSHI Scores," the symbol was missing in the following sentence:

However, scores were marginally higher among participants with a Kessler 6-item psychological distress scale (K6) score13.

This has been corrected to:

However, scores were marginally higher among participants with a Kessler 6-item psychological distress scale (K6) score $\geq 13$.

The correction will appear in the online version of the paper on the JMIR Publications website on March 5, 2021, together with the publication of this correction notice. Because this was made after submission to PubMed, PubMed Central, and other full-text repositories, the corrected article has also been resubmitted to those repositories. 
This is a non-peer-reviewed article. Submitted 02.03.21; accepted 03.03.21; published 05.03.21.

Please cite as:

Jackman KMP, Kane J, Kharrazi H, Johnson RM, Latkin C

Correction: Using the Patient Portal Sexual Health Instrument in Surveys and Patient Questionnaires Among Sexual Minority Men

in the United States: Cross-sectional Psychometric Validation Study

$J$ Med Internet Res 2021;23(3):e28358

URL: https://www.jmir.org/2021/3/e28358

doi: $10.2196 / 28358$

PMID: $\underline{3667179}$

CKevon-Mark P Jackman, Jeremy Kane, Hadi Kharrazi, Renee M Johnson, Carl Latkin. Originally published in the Journal of Medical Internet Research (http://www.jmir.org), 05.03.2021. This is an open-access article distributed under the terms of the Creative Commons Attribution License (https://creativecommons.org/licenses/by/4.0/), which permits unrestricted use, distribution, and reproduction in any medium, provided the original work, first published in the Journal of Medical Internet Research, is properly cited. The complete bibliographic information, a link to the original publication on http://www.jmir.org/, as well as this copyright and license information must be included. 